\title{
Intermediate-Node Initiated Reservation (IIR): A New Signaling Scheme for Wavelength-Routed Networks
}

\author{
Kejie Lu, Student Member, IEEE, Jason P. Jue, Member, IEEE, Gaoxi Xiao, Member, IEEE, \\ Imrich Chlamtac, Fellow, IEEE, and Timucin Ozugur, Member, IEEE
}

\begin{abstract}
A problem of many distributed lightpath provisioning schemes is wavelength contention, which occurs when a connection request attempts to reserve a wavelength channel that is no longer available. This situation results from the lack of updated global link-state information at every node. In networks with highly dynamic traffic loads, wavelength contention may seriously degrade the network performance. To overcome this problem, we propose a new framework for distributed signaling and introduce a class of schemes referred to as intermediate-node initiated reservation. In the new scheme, reservations may be initiated at any set of nodes along the route; in contrast, reservations can only be initiated by the destination node in the classic destination initiated reservation (DIR) scheme. As a result, the possibility of having outdated information due to propagation delay will be significantly lowered. Specifically, we consider two schemes within this framework, for networks with no wavelength conversion and for networks with sparse wavelength conversion, respectively. Theoretical and simulation results show that, compared with the classic DIR scheme, the new schemes can significantly improve the network blocking performance. The accuracy of the analytical models is also confirmed by extensive numerical simulations.
\end{abstract}

Index Terms-Blocking probability, distributed lightpath provisioning, signaling, wavelength-division multiplexing (WDM), wavelength-routed network.

\section{INTRODUCTION}

W AVELENGTH-DIVISION multiplexing (WDM) technology has been progressing steadily, with existing systems capable of providing more than $1-\mathrm{Tb} / \mathrm{s}$ bandwidth on a single optical fiber. To fully utilize these high data rates, all-optical connections, or lightpath [1], can be established between source and destination nodes. Lightpath-based optical networks are generally referred to as wavelength-routed networks. Wavelength-routed networks are expected to provide better flexibility and better network resource efficiency than existing point-topoint WDM networks.

In legacy backbone networks, traffic is generally static, with connections remaining in the network semipermanently. How-

Manuscript received January 2, 2003; revised June 16, 2003. This paper was supported in part by Alcatel.

K. Lu, J. P. Jue, and I. Chlamtac are with the Center for Advanced Telecommunications Systems and Services (CATSS), The University of Texas at Dallas, Richardson, TX 75083 USA (e-mail: lukejie@utdallas.edu; jjue@utdallas.edu; chlamtac@utdallas.edu).

G. Xiao is with the School of Electrical and Electronic Engineering, Nanyang Technological University, 639798 Singapore (e-mail: EGXXiao@ntu.edu.sg).

T. Ozugur is with Alcatel Research and Innovation Center, Plano, TX 75075

USA (e-mail: tim.ozugur@alcatel.com).

Digital Object Identifier 10.1109/JSAC.2003.816625 ever, in next-generation optical networks, especially in the optical Internet, data traffic is expected to be more dynamic. In the extreme case, such as in optical burst switched (OBS) networks [4], it is expected that the connection requests will arrive at very high rates, and that the average duration of each connection will only be several tens or hundreds of milliseconds [5]. To cope with these new data traffic loads, the development of dynamic lightpath provisioning schemes will become increasingly important [1]-[3].

Dynamic lightpath provisioning can be handled in a centralized manner, in which every decision is made by a central controller, or it can be handled in a distributed manner, in which decisions are distributed to different network nodes. While centralized schemes may perform more efficiently for small networks with static traffic loads, distributed schemes may be more appropriate for large optical networks with bursty Internet traffic loads. Distributed schemes have been proposed and are now being standardized within the framework of generalized multiprotocol label switching (GMPLS) [6]. In this paper, we focus on distributed control schemes.

In a GMPLS-based network, routing protocols such as open shortest path first with traffic engineering (OSPF-TE) are used to exchange routing information, including topology and resource availability among nodes. Based on the global link-state information, a route is calculated by using a constraint-based routing algorithm when a connection request is received. Once the route is determined, a signaling scheme is responsible for establishing the lightpath. Candidates for the signaling protocols within the GMPLS framework include Resource reSerVation Protocol with Traffic Engineering (RSVP-TE) [7] and constraint-based routing label distribution protocol (CR-LDP) [8]. Regardless of which signaling protocol is used, there is no guarantee that the updated global information with respect to wavelength availability on each link will be available in a distributed environment. Link-state information may become outdated because the update messages are broadcasted only periodically and also because it takes some time for the updates to propagate to a node. To solve this problem, several reservation schemes have been studied.

A well-known distributed signaling scheme is the destination initiated reservation (DIR) method [9]. In the DIR method, a connection request is forwarded from the source to the destination, collecting the wavelength availability information of every link along the route. Based on this information, the destination node will select an available wavelength along the path (if such 


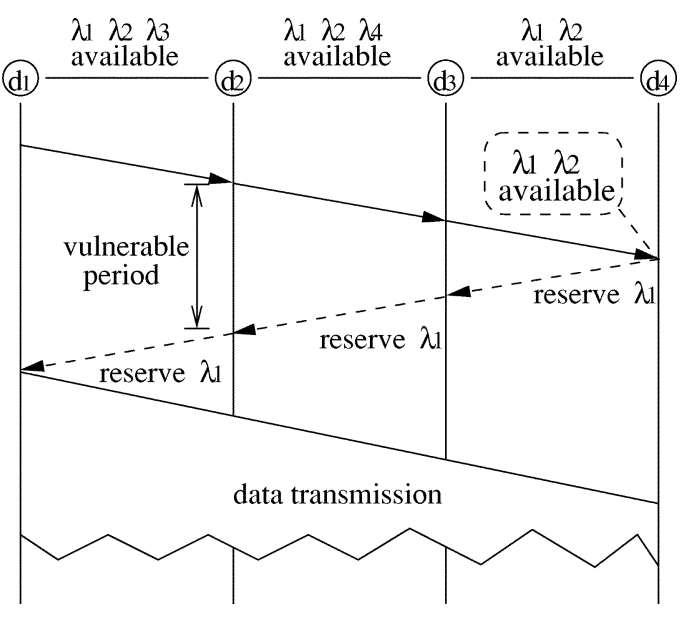

(a)

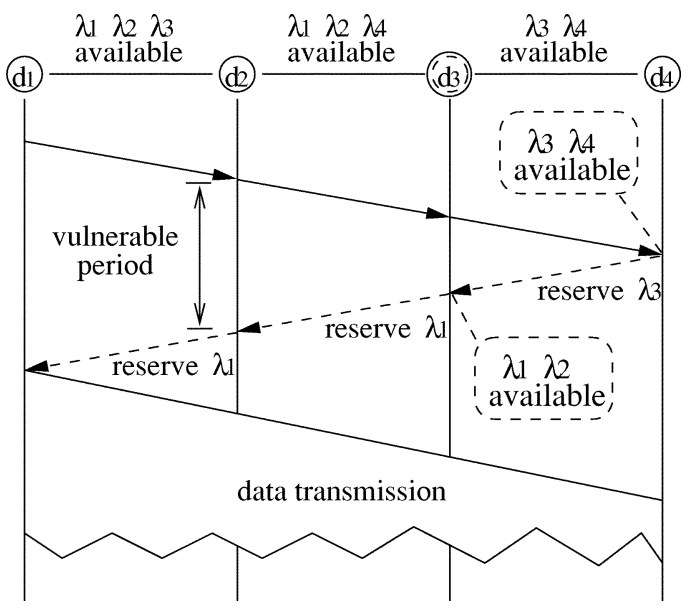

(b)

Fig. 1. Examples of the DIR scheme. (a) Networks with no wavelength conversion. (b) Networks with sparse wavelength conversion.

is available) and send a reservation request back to the source node to reserve the selected wavelength.

Fig. 1(a) shows an example of the DIR method in a network with no wavelength conversion. In a network without wavelength conversion, a connection request will be rejected if a common wavelength cannot be found along the route. This constraint is known as the wavelength continuity constraint [1]. From Fig. 1(a), we can also observe that, if the wavelength continuity constraint does not exist, a connection may have a greater chance of being successfully established. Elimination of the wavelength continuity constraint can be realized by installing wavelength converters on every node. However, from a practical point of view, this approach is difficult to implement due to the high cost of wavelength converters [10]. Another solution is to install wavelength converters only on a subset of nodes, namely the sparse conversion [11] solution. Fig. 1(b) shows an example of the DIR method in networks with sparse wavelength conversion, where only node $d_{3}$ has wavelength conversion capability.

Another well-known signaling scheme is the source initiated reservation (SIR) method [9], where wavelength resources are reserved as a control messages traverses along the forward path to the destination. In the SIR scheme, the number of wavelengths to be reserved generally depends on how much informa- tion is available to every node along the path. When the source node has the complete link-state information, it may try to reserve only a single available wavelength along the path. However, for most of the dynamic traffic conditions where up-to-date global information is not available, the source node may reserve all the available wavelengths along the route [9] or a group of wavelengths on each hop of the route [12]. Generally, the DIR scheme will outperform the SIR scheme in networks with no wavelength conversion [9].

It has been shown that, for the DIR scheme under bursty traffic loads, connection blocking is primarily caused by outdated information [15], [16] (i.e., wavelength contention). Specifically, when a reservation request reaches a link intending to reserve a wavelength, it may find that the wavelength has been reserved by another reservation request that arrived earlier. It also has been shown that this kind of blocking increases significantly with respect to the vulnerable period, which is the delay between the moment that the link-state information is collected and the moment that the reservation request arrives (see Fig. 1). In the SIR scheme, on the other hand, reserving a greater amount of resources for the current connection request can increase the probability that the current request will be accepted; however, reserving too many network resources may block some other simultaneous connection requests, causing an over-reservation problem.

To deal with the outdated-information problem of the DIR scheme and the over-reservation problem of the SIR scheme, we present a new framework for distributed signaling schemes and a new signaling approach referred to as intermediate-node initiated reservation (IIR). The main idea is to allow the reservation to be initiated by a set of intermediate nodes before the connection request arrives to the destination node. This technique will reduce the amount of over-reservation and will reduce the blocking caused by outdated information due to a shorter vulnerable period. We first investigate two signaling schemes, one for networks with no wavelength conversion and the other for networks with sparse wavelength conversion. Studies of these cases demonstrate the benefits of the new schemes, and also provide some insight into the new framework.

We study the performance of the simple cases by extensive numerical simulations and theoretical analysis. A key measurement of performance in these studies is the connection blocking probability, the probability that an arriving connection request will be rejected. Blocking probability in wavelength-routed optical networks has been studied analytically in a number of previous works. While most previous research (e.g., [11], [13], and [14]) considers only centralized cases, distributed schemes are studied in [15] and [16]. In [15], analytical models are developed to evaluate the blocking probability characteristics of various SIR and DIR schemes. The models consider blocking of connections due to insufficient resources, as well as blocking due to outdated information. More accurate models are developed in [16] to measure the blocking probability in networks with no wavelength conversion. In this paper, we further extend the analytical model to handle the case in which reservations could be initiated by intermediate nodes, and the case of networks with or without sparse wavelength conversion. We also analyze the control complexity of the new methods, since control overhead 
is an important concern of virtually all distributed lightpath provisioning schemes.

The rest of this paper is organized as follows. In Section II, we provide the general framework for the IIR scheme. We then propose two simple schemes within the framework, for networks with no conversion and with sparse conversion. Analytical models are developed in Section III to evaluate the blocking performance of the proposed scheme. Numerical results and discussions are presented in Section IV. Section V concludes the paper.

\section{INTERMEDIATE-NODE INITIATED RESERVATION SCHEME}

In this section, we first propose the general framework for the IIR scheme. We then describe two simple signaling methods within the framework for networks with no conversion and for networks with sparse conversion. The control complexity of each of the two methods is also discussed in this section.

We now provide a formal definition of the IIR scheme. Suppose route $R$ consists of a sequence of nodes, from source to destination

$$
\left\{d_{1}^{R}, d_{2}^{R}, \ldots, d_{N^{R}}^{R}\right\}
$$

where $N^{R}$ denotes the total number of nodes in route $R$. We define the following parameters.

$I^{R} \quad$ number of nodes on route $R$ from which reservations can be initiated, $1 \leq I^{R} \leq N^{R}$;

$S^{R} \quad$ set of nodes on route $R$ from which reservations can be initiated

$$
S^{R}=\left\{s_{1}^{R}, s_{2}^{R}, \ldots, s_{I^{R}}^{R}\right\}
$$

$\delta_{i}^{R} \quad$ for each node $s_{i}^{R} \in S^{R}$, we define $\delta_{i}^{R}$ as the direction of the reservation initiated by the node. $\delta_{i}^{R}$ can be "forward" (i.e., direction from the source to the destination), "backward" (i.e., direction from the destination to the source), or "bidirectional;"

$W_{i}^{R}$ at any node $s_{i}^{R} \in S^{R}, W_{i}^{R}$ denotes the set of wavelengths to be reserved.

For a given route $R, I^{R}, S^{R}$, and $\delta_{i}^{R}$ and $W_{i}^{R}$ for each $s_{i}^{R} \in$ $S^{R}$ can be specified in a static manner, in a dynamic manner by the source node, or in a dynamic manner as the connection request is traversing route $R$.

Note that, if $I^{R}=1, s_{1}^{R}=d_{1}^{R}$, and $\delta_{1}^{R}=$ "forward," then a reservation message will be sent in the forward direction by the source node, which is equivalent to SIR. ${ }^{1}$ On the other hand, if $I^{R}=1, s_{1}^{R}=d_{N^{R}}^{R}, \delta_{1}^{R}=$ "backward," and $\left|W_{1}^{R}\right|=1$, then a reservation message will be sent in the backward direction by the destination, which is equivalent to DIR. Thus, the proposed signaling scheme will perform at least as good as SIR and DIR if the parameters of IIR are allowed to adapt to various traffic conditions.

The generalized protocols may include algorithms for determining the optimal selection of the nodes for initiating

${ }^{1} W_{1}^{R}$ depends on different SIR implementations. For example, the greedy SIR may reserve all available wavelengths. the reservations and the algorithms for determining the best number of wavelengths to reserve. The choice of the parameters in the optimization process could be based on various requirements and properties of the specific connection request, such as quality of service requirements, priorities, hop distance, and other constraints. Therefore, by connection-specific provisioning and proper optimization, the protocol can provide differentiated levels of service and also ensure fairness for connections of different lengths. We now propose two static IIR implementations for networks with no wavelength conversion and for networks with sparse wavelength conversion.

\section{A. IIR for Networks With No Wavelength Conversion}

To design an appropriate IIR method, one important issue is to determine the $S^{R}$ for each route. The choice of $S^{R}$ may vary depending on the route. For single-hop routes, the destination node can be selected to initiate the reservation request because the vulnerable period is always zero. Therefore, only one wavelength is needed since there is no blocking due to outdated information.

For multihop routes, the wavelength continuity constraint must be satisfied in networks with no wavelength conversion. In other words, the same wavelength must be reserved along the route. Intuitively, if the reservation is initiated at a node that does not have sufficient link-state information on the path, then multiple wavelengths must be reserved. For example, in the SIR scheme, multiple wavelengths must be reserved in order to ensure the successful lightpath establishment. However, reserving too many wavelengths may block other simultaneous connections, causing an over-reservation problem. On the other hand, in the DIR scheme, only a single wavelength is reserved. While reserving only a single wavelength may cause blocking due to outdated information, generally the DIR scheme still outperforms the SIR scheme since blocking caused by over-reservation is more significant.

To avoid the over-reservation problem, a simple approach is to design an IIR method such that only one wavelength is reserved. Given this constraint, it is important to choose nodes in $S^{R}$ that have adequate link-state information of the entire path. One possible candidate is the destination node. However, as we can observe from Fig. 1(a), at the upstream node of each link, the wavelength usage information of the link is also available, though this information may not always be accurate because of the propagation delay between the two end nodes of the link. ${ }^{2}$ Consequently, when a forward message arrives at the upstream node of the last link, the wavelength availability information of the entire route is available, though the information of the last link could be slightly outdated. Based on this observation, we develop a simple scheme within the IIR framework in which the upstream node of the last link initiates a reservation request, referred to as the fast reservation request, back to the source node.

An example for this IIR implementation is shown in Fig. 2(a). Comparing this example with Fig. 1(a), we observe that, if the

\footnotetext{
${ }^{2}$ In GMPLS context, the downstream node of a link is responsible for the resource assignment for the ingress link. Ingress or any upstream node can restrict the labels that may be selected by downstream node by using the suggested label object.
} 


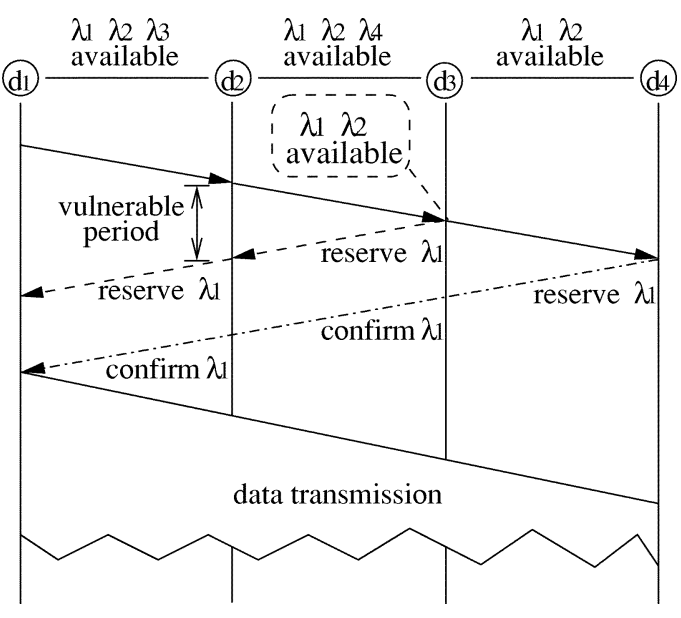

(a)

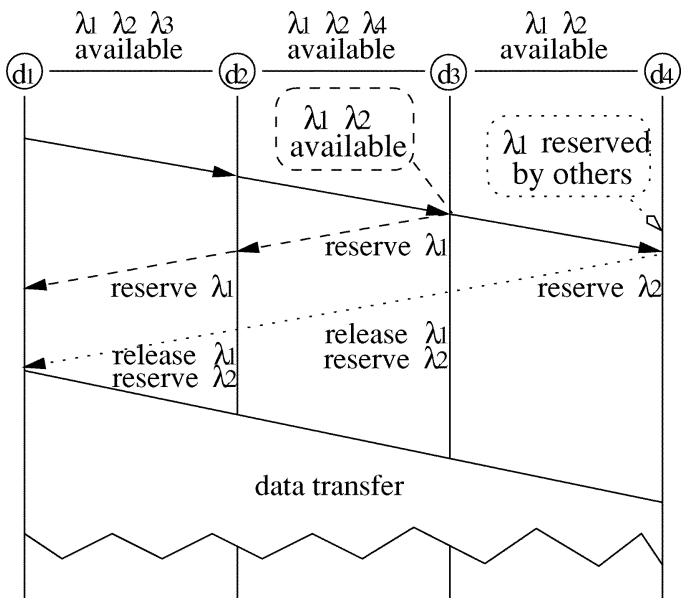

(b)

Fig. 2. Examples of the IIR scheme for networks with no conversion. (a) Without last link conflict and (b) with last link conflict.

reservation on the destination node is successful, then the vulnerable period at node $d_{2}$ and $d_{3}$ can be reduced by the round trip propagation delay on the last link. From Fig. 2(b), we can also observe that the selected wavelength may have been reserved by another reservation request that arrives at the destination node earlier. To handle this last link conflict problem, the destination node is allowed to initiate another reservation, provided that there exists another available wavelength along the path. Note that, if the destination reserves another wavelength in the backward direction, the wavelength previously selected must be released [see Fig. 2(b)].

We now provide a formal description of the above static IIR.

- If $N^{R}=2$, which means the connection has only one hop, we let

$$
\left\{\begin{array}{l}
I^{R}=1 \\
s_{1}^{R}=d_{2}^{R} \\
\delta_{1}^{R}=\text { "backward" }
\end{array}\right.
$$

and we let $W_{1}^{R}$ be a single wavelength which is randomly selected from the available wavelengths (if any) along the path. In this case, the IIR scheme performs the same as the DIR scheme.
- If $N^{R}>2$, we let

$$
\left\{\begin{array}{l}
I^{R}=2 \\
s_{1}^{R}=d_{N^{R}-1}^{R} \\
\delta_{1}^{R}=\text { "bidirectional" } \\
s_{2}^{R}=d_{N^{R}}^{R} \\
\delta_{2}^{R}=\text { "backward" }
\end{array}\right.
$$

and we let $W_{1}^{R}$ be a randomly selected available wavelength. If $W_{1}^{R}$ is still available when the connection request reaches the destination node, then $W_{2}^{R}=W_{1}^{R}$; otherwise, $W_{2}^{R}$ will be another randomly selected available wavelength.

Although the vulnerable period is reduced by only the propagation delay on the last link, this reduction can nevertheless significantly improve the network performance in typical mediumsized optical networks, especially under highly dynamic and bursty traffic loads.

To support the proposed IIR scheme in networks with no wavelength conversion, current signaling protocols need to be extended. Compared with the DIR scheme, the IIR scheme has the following requirements.

1) Each node maintains the link-state information of its egress links.

2) The fast reservation message must be distinguished from the normal reservation message initiated by the destination node. Specifically,

- once a fast reservation message is received, the source node will update the link state of the egress link. However, the source node will not begin the data transmission until it receives a confirmation from the destination node;

- once a normal reservation message is received, and if the wavelength indicated in this message is not the same as that indicated by the fast reservation message, then the node must release the wavelength previously reserved.

3) If a normal reservation request reserving a different wavelength also fails at any intermediate node, then two release requests may have to be sent out (one in the forward direction and the other in the backward direction) to release two different reserved wavelengths, respectively.

From the discussions above, we see that the proposed signaling scheme needs more control intelligence than the classic DIR scheme. However, the extra control can still be realized within the GMPLS framework by extending signaling protocols such as CR-LDP and RSVP-TE. The detailed implementations are beyond the scope of this paper. The additional control overhead is caused by the fast reservation requests, as well as the release process that are required when a conflict occurs on the last link (to release the previously reserved wavelength). In our simulation, we will show that the extra control overhead is generally not significant.

\section{B. IIR for Networks With Sparse Wavelength Conversion}

In networks with sparse wavelength conversion, the wavelength continuity constraint is relaxed. We observe from 


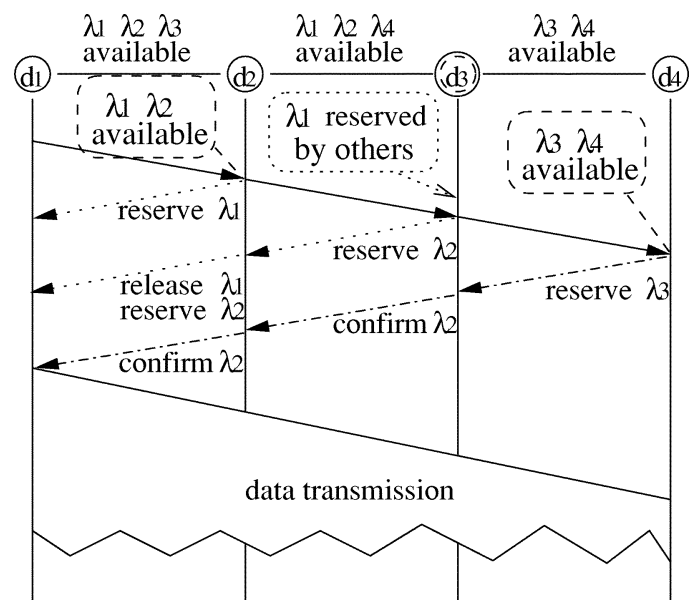

Fig. 3. Example of the IIR scheme for network with sparse wavelength conversion.

Fig. 1(b) that a route can be separated into several segments such that the end nodes of each segment are the source node, the destination node, or the intermediate nodes with conversion capabilities. On different segments, we may reserve different wavelengths, as shown in Fig. 1(b).

Since the wavelength continuity constraint only holds within one segment, the reservations within each segment, referred to as segment reservations [17], can be viewed as largely independent from each other. Therefore, the simple scheme we proposed can be further extended such that a fast segment reservation is initiated at the upstream node of the last link of each segment if the segment consists of two or more links, and a segment reservation message is initiated at the downstream node of each segment except the last segment. Finally, the normal reservation message initiated in the last segment, i.e., by the destination node, will go through the entire route to inform the source node to start the data transmission. If the reservation is not successful, then the normal reservation message will reach the intermediate node where backward blocking occurred and then trigger proper release operations.

Fig. 3 illustrates the proposed IIR scheme for networks with sparse wavelength conversion. We observe that, by exploiting the sparse conversion capability, the vulnerable period can be drastically reduced. For example, comparing Fig. 1(b) with Fig. 3, we see that the vulnerable period on node $d_{2}$ is reduced from the round-trip propagation delay between $d_{2}$ to $d_{4}$ to zero, if there is no conflict on the last link. Consequently, blocking due to outdated information is significantly lowered.

To support the proposed IIR scheme in networks with sparse conversion, the control intelligence required at each node remains nearly the same as that in the nonconversion networks, except that each node must be able to distinguish the segment reservation messages from other reservation messages.

\section{THEORETICAL ANALYSIS}

Compared with the classic DIR scheme, IIR schemes are expected to have lower blocking probability in the backward direction, since the vulnerable period is reduced. However, network capacity would be reserved for longer time before the transmission actually begins, which may increase the blocking proba- bilities in the forward direction. To evaluate the performance of the IIR framework, we develop accurate analytical models by taking both of the above effects into consideration. We develop a model for the DIR scheme and another the simple IIR scheme.

Since a network with no wavelength conversion can be viewed as a special case of a network with sparse wavelength conversion, we only consider the sparse conversion cases. To simplify the analysis, we make the following assumptions.

- The network is composed of $J$ links connected in an arbitrary topology.

- Each link is composed of $C$ wavelength channels.

- Wavelength conversion is available only at a certain given set of nodes.

- The connection requests for each pair of source-destination nodes arrive from a Poisson process with an arrival rate $\lambda_{R}$, where $R$ denotes the fixed route between the two nodes.

- Connection holding time is exponentially distributed with a parameter $\mu$.

- Random wavelength assignment policy is adopted.

\section{A. Framework}

The framework of the analytical models is similar to that in [16]. Specifically, we define the link state as the state of the wavelength channels on a link when a connection request reaches the downstream node of the link. A wavelength channel on any link can be in one of the following three states: 1) free; 2) reserved, yet with no data transmission; and 3) occupied by data transmission. We further denote that a channel is busy if it is in state 3); otherwise, it is idle.

Let $X_{j}$ be the number of idle wavelength channels on link $j$, and let $q_{j}(m)$ be the probability that $X_{j}=m$. We further assume that when there are $m$ idle wavelength channels on link $j$, the interarrival time of connection requests is exponentially distributed with a parameter $\lambda_{j}(m)$. Therefore, the state of each link can be characterized by a state-dependent $\mathrm{M} / \mathrm{M} / \mathrm{C} / \mathrm{C}$ model (as shown in Fig. 4)

$$
\begin{aligned}
q_{j}(m) & =q_{j}(0) \cdot \mu^{m} \cdot \prod_{k=1}^{m} \frac{C-k+1}{\lambda_{j}(k)} \quad m=1,2, \ldots, C \\
q_{j}(0) & =\left[1+\sum_{m=1}^{C}\left(\mu^{m} \cdot \prod_{k=1}^{m} \frac{C-k+1}{\lambda_{j}(k)}\right)\right]^{-1}
\end{aligned}
$$

Using the reduced-load approximation algorithm, the framework could be described as follows.

1) Initiate $\lambda_{j}(m), j=1,2, \ldots, J$ as follows: Let $\lambda_{j}(0)=$ 0 and $\lambda_{j}(m)=\sum_{R: j \in R} \lambda_{R}, m=1,2, \ldots, C$.

2) Calculate $q_{j}(m), j=1,2, \ldots, J$ through (1).

3) Calculate the blocking probability of $R$ as

$$
B_{R}=1-V_{R}=1-V_{R}^{F} \times V_{R}^{B}
$$

where $V_{R}$ denotes the probability that a reservation is successful along the route $R, V_{R}^{F}$ denotes the probability that a reservation is successful along the forward direction, and $V_{R}^{B}$ denotes the conditional probability that a reservation is successful along the backward direction given that 


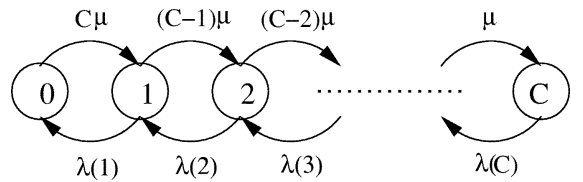

Fig. 4. State dependent link-state model.

it was successful on the forward direction. If, for every route $R, B_{R}$ has been convergent, then stop; otherwise, go to Step 4.

4) Calculate $\lambda_{j}(m), j=1,2, \ldots, J$ as follows:

$\lambda_{j}(m)=\sum_{R: j \in R} \lambda_{R, j}(m) \triangleq \sum_{R: j \in R} \lambda_{R} \cdot V_{R \mid X_{j}=m}$

where $\lambda_{R, j}(m)$ denotes the arrival rate of those connection requests for route $R$ which are finally successfully accepted, given that the state of link $j$ is $m$. Go to Step 2. We next discuss the calculation of $V_{R}^{F}$ and $V_{R}^{B}$.

\section{B. Blocking in the Forward Direction}

A connection request can successfully reach the destination node if and only if, in all segments of the route, there is at least one available wavelength. Therefore

$$
V_{R}^{F}=\prod_{s=1}^{S_{R}} V_{s}^{F}
$$

where $S_{R}$ denotes the number of segments in the route $R$. Here we assume that successful probabilities on different segments are independent. Within each segment, we use the model developed in [16], while the calculations of the following two steady-state probabilities must be modified to reflect the blocking caused by channels in the reserved state:

$f_{i, j} \quad$ denotes the conditional probability that a given set of $i$ channels are free on link $j$, given that these $i$ channels are idle;

$f_{i, j \mid i, j^{\prime}} \quad$ denotes the conditional probability that a given set of $i$ wavelength channels are free on link $j$, given that these $i$ channels are idle and that they were free on the upstream link $j^{\prime}$ when the forward message arrived.

The calculations of $f_{i, j}$ and $f_{i, j \mid i, j^{\prime}}$ can be found in Appendix A.

\section{Blocking in the Backward Direction}

Similar to the forward blocking analysis, a reservation request is successful if and only if it is successful in all segments. Therefore, based on the same independent assumption as (4), we have

$$
V_{R}^{B}=\prod_{s=1}^{S_{R}} V_{s}^{B}
$$

For the DIR scheme, we let $w_{s}$ denote the probability that the reservation request for route $R$ is not blocked at the downstream node of segment $s$; and $w_{j, j^{\prime \prime}}$ denote the probability that $R$ is not blocked at $j$ given that the downstream link $j^{\prime \prime}$ is not on the route of the interfering reservation request. Therefore

$$
V_{s}^{B}= \begin{cases}w_{s}(t), & L_{s}=1 \\ w_{s}(t) \times \prod_{j=1}^{L_{s}-1} w_{j, j^{\prime \prime}}(t), & L_{s}>1\end{cases}
$$

where parameter $t$ denotes the vulnerable period for a given link and $L_{s}$ denotes the number of links in the current segment. For the classic DIR scheme, the vulnerable period on link $j$ equals the round trip propagation delay of the downstream node of link $j$ and the destination node. For calculations of $w_{s}$ and $w_{j, j^{\prime \prime}}$, refer to [17].

For the IIR scheme, obviously, if $L_{s}=1$, then $V_{s}^{B}=1$ since the vulnerable period is always 0 . To calculate $V_{s}^{B}$ for $L_{s}>1$, we define two variables:

$y_{s} \quad$ probability that the wavelength selected at upstream node of link $j_{R}^{s}$ (the last link of segment $s$ ) is not occupied by another connection when the connection request for $R$ arrives at the downstream node of $j_{R}^{s}$;

$z_{s} \quad$ conditional probability that at least one channel is available when the forward message arrives at the downstream node of $j_{R}^{s}$, given that the connection request successfully reaches the upstream node of $j_{R}^{s}$ but the wavelength selected by that node has been reserved by another reservation request when the Therefore forward message reaches the downstream node of $j_{R}^{s}$.

$$
\begin{aligned}
V_{s}^{B}=y_{s}\left(t_{1}\right) \times \prod_{j=1}^{L_{s}-1} w_{j, j^{\prime \prime}}\left(t_{2}\right) & \\
& +\left(1-y_{s}\left(t_{1}\right)\right) \times z_{s} \times \prod_{j=1}^{L_{s}-1} w_{j, j^{\prime \prime}}\left(t_{3}\right)
\end{aligned}
$$

where vulnerable periods are defined as

$t_{1}$ round trip propagation delay between the two end nodes of link $j_{s}^{R}$;

$t_{2}$ round trip propagation delay between the downstream node of link $j$ and the upstream node of link $j_{s}^{R}$;

$t_{3} \quad$ round trip propagation delay between the downstream node of link $j$ and the downstream node of link $j_{s}^{R}$.

We can observe from (7) that the last link conflict is reflected in the analytical model. The calculations of $y_{s}$ can be found in Appendix $\mathrm{B}$ and the calculation of $z_{s}$ is similar to the calculation of $w_{s}$.

\section{State-Dependent Arrival Rate} [17]

The calculations of the $V_{R \mid X_{j}=m}$ remains unchanged from

\section{NUMERICAL RESUlTS}

We verify the blocking performance of the simple signaling methods within the IIR framework, as well as the accuracy of the proposed analytical models by extensive simulations. In our simulations, we assume that

- the traffic pattern is uniform, i.e., the arrival rate of connection requests between each pair of source-destination nodes is identical; 


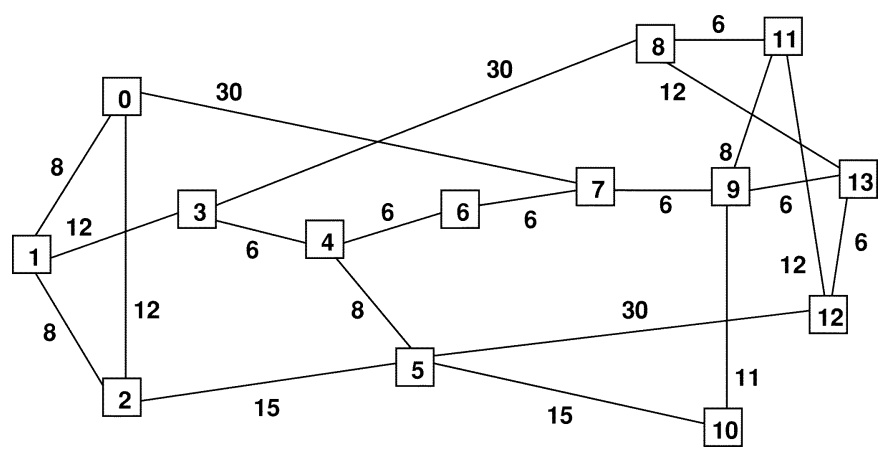

Fig. 5. Network topology of NSFNet.

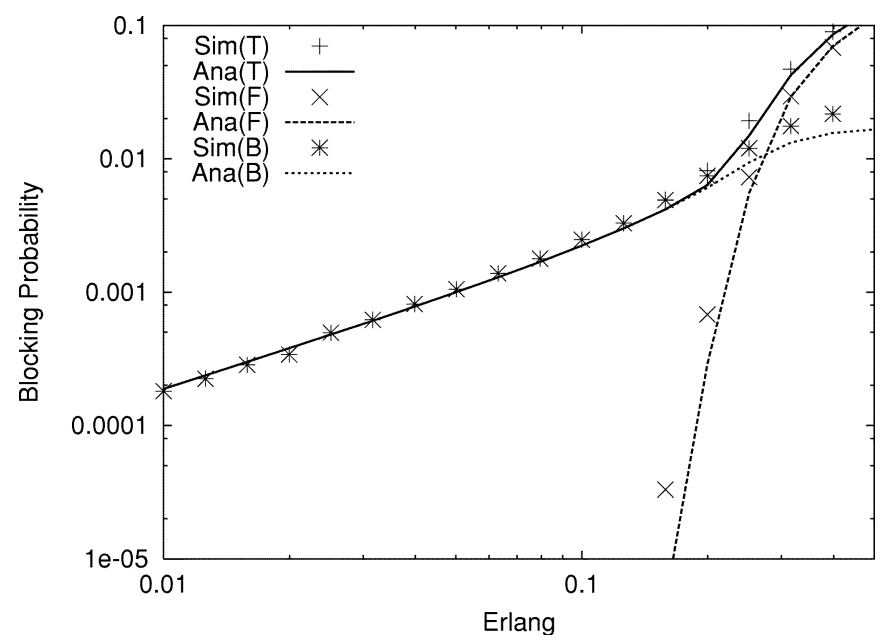

(a)

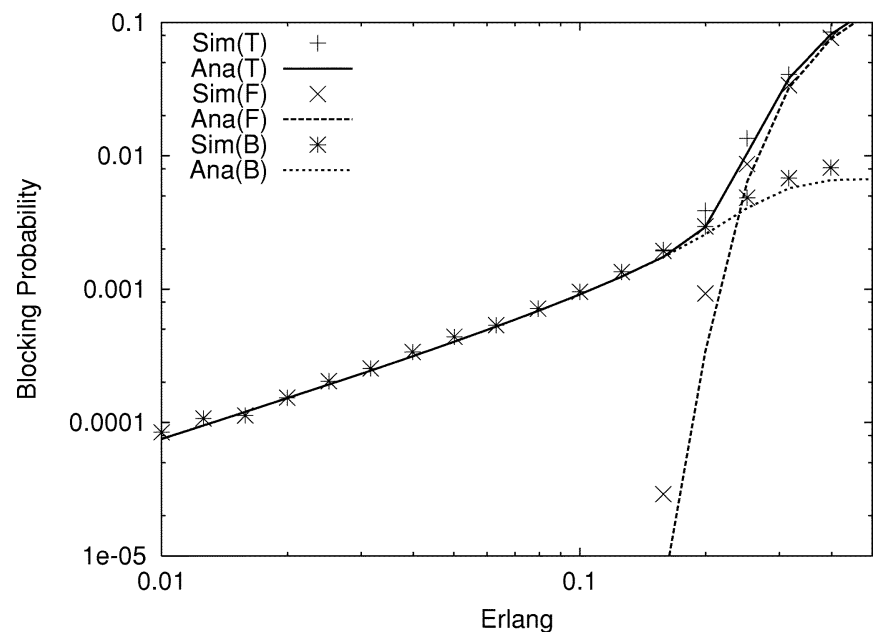

(b)

Fig. 6. Blocking of different signaling schemes in NSFNet with no wavelength conversion $(\mathrm{C}=32, \mathrm{D}=1 \mathrm{~s})$. (a) DIR. (b) IIR.

- fixed shortest-path routing is used between each pair of source-destination nodes.

We conduct our simulations on three different network topologies.

1) The NSFNet topology, shown in Fig. 5, where the numbers next to each link denote the physical length in $100 \mathrm{~s}$ of kilometers. For the sparse conversion case, we assume that wavelength conversion is available on nodes $3,5,7$, and 9.

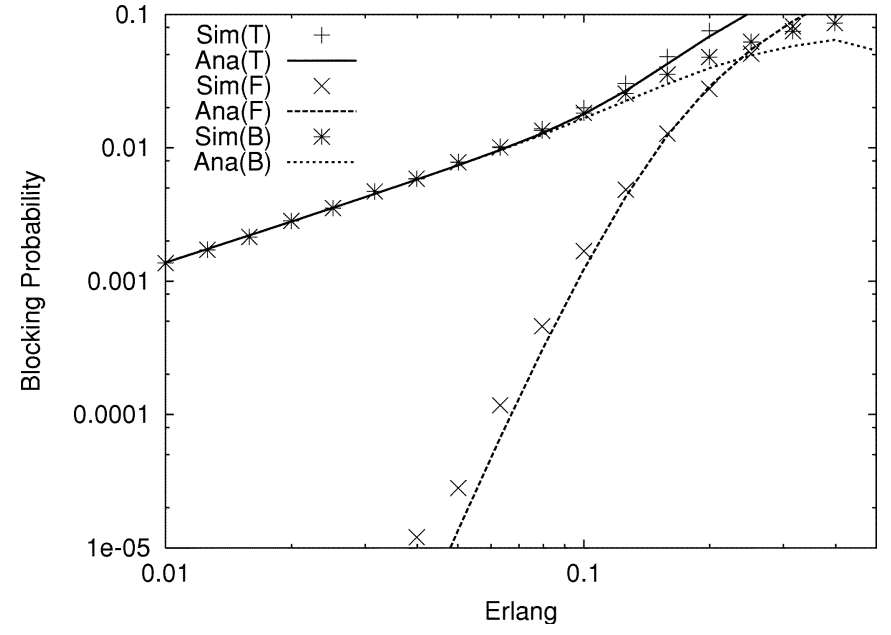

(a)

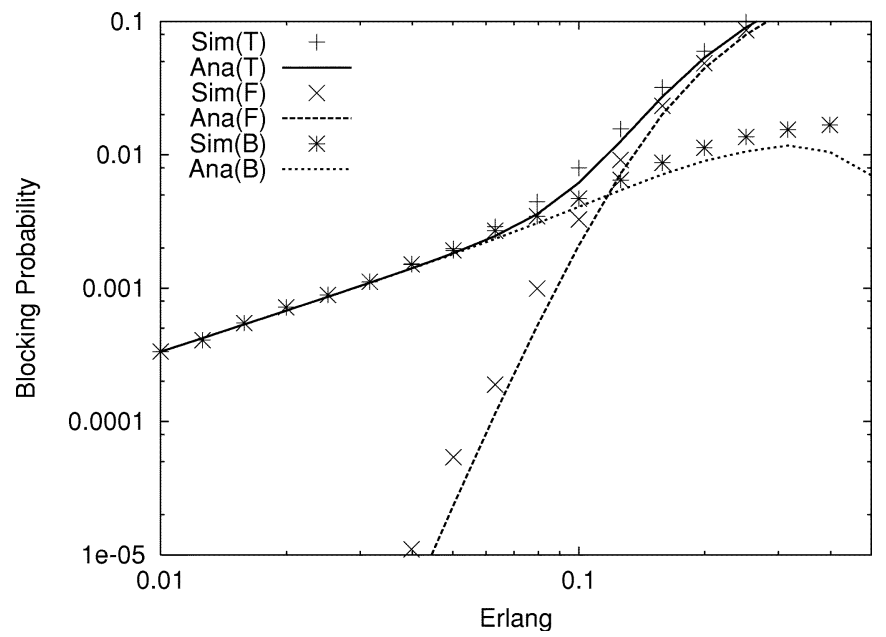

(b)

Fig. 7. Blocking of different signaling schemes in NSFNet parse wavelength conversion (C=8 and D $=0.1 \mathrm{~s}$ ). (a) DIR. (b) IIR.

2) A 12-node ring topology where each link is $10 \mathrm{~km}$ in length. For the sparse conversion case, we assume that four wavelength-conversion nodes are evenly distributed in the network.

3) A 25-node randomly generated network topology where the average nodal degree is 4 and the average length of each link is $200 \mathrm{~km}$. For the sparse conversion case, we assume that five randomly selected nodes have wavelength conversion capability.

For all three topologies, we assume that each link consists of two directional fibers with opposite directions, with the same number of wavelength channels per fiber. In all the simulation results, the traffic load, measured in Erlang, denotes the normalized traffic loads originating from each node; and $D(D=1 / \mu)$ denotes the average holding time of every connection.

Figs. 6 and 7 show the high accuracy of our analytical models for both the classic DIR scheme and the new IIR scheme, with and without sparse wavelength conversion. These results confirm that the analytical model correctly reflects the effect of parameters such as a shorter vulnerable period and a longer duration in the reserved state on network performance. We observe that, under light traffic loads, traffic blocking primarily takes place in the backward direction, and is caused by outdated in- 


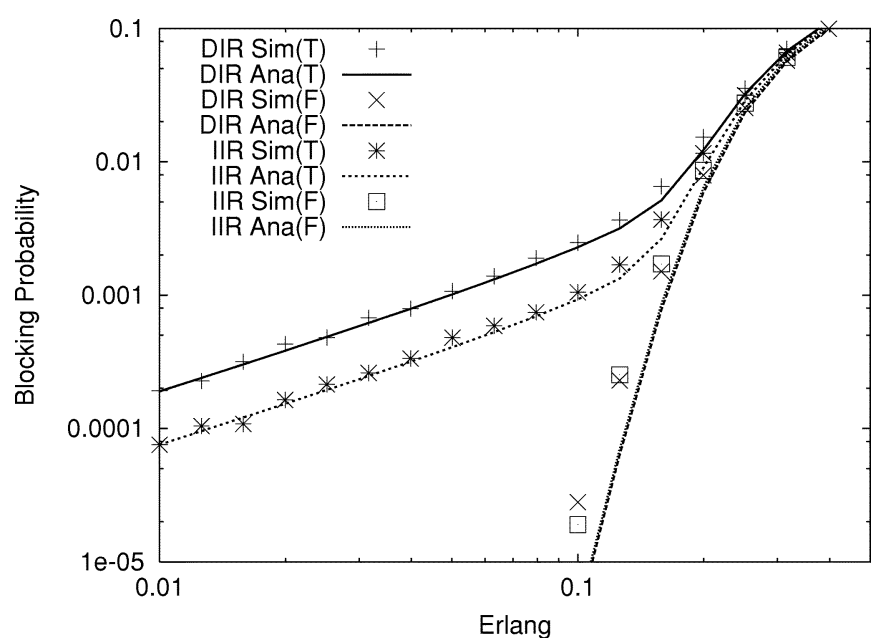

(a)

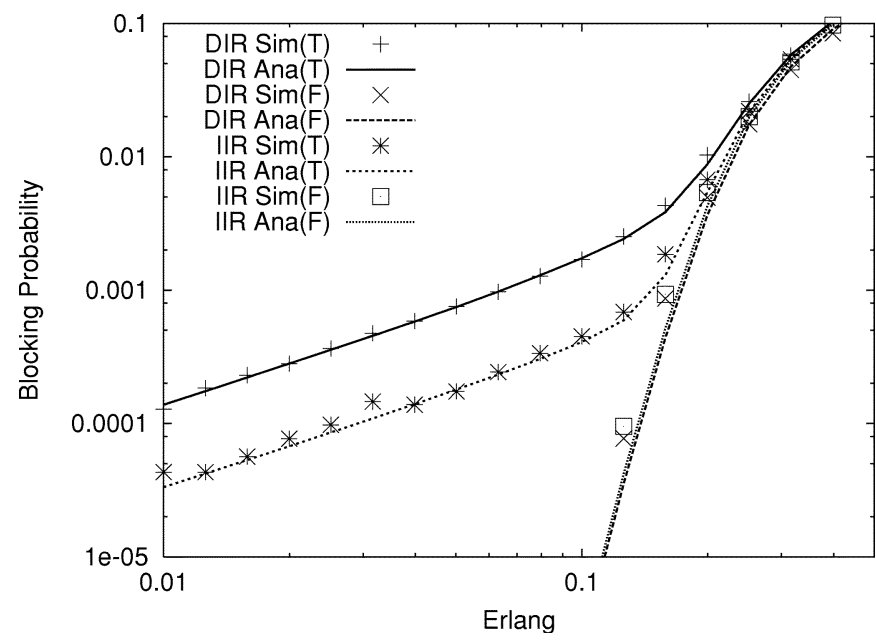

(b)

Fig. 8. DIR versus IIR scheme in NSFNet $(C=16, D=1 \mathrm{~s}$ ). (a) No conversion. (b) Sparse conversion.

formation; whereas under heavy traffic loads, blocking occurs primarily in the forward direction and is due to insufficient network capacity.

Fig. 8 compares the performance of DIR and IIR schemes under different network scenarios. We observe that, although the IIR scheme slightly increases the forward blocking, it significantly reduces the backward blocking. As a result, the overall blocking probability is significantly lowered, especially under light traffic loads. Under very heavy traffic loads, the forward blocking is dominant, thus, the blocking probabilities of the two cases is nearly the same. By comparing Fig. 8(a) and (b), we also see that, with sparse wavelength conversion, the improvements that could be achieved by using the IIR scheme become more significant.

In Fig. 9, we compare the performance of the DIR and the IIR schemes for the 12-node ring topology and the 25-node randomly generated network topology, respectively. Here, $C=16$ for both networks, and sparse conversion is used. We observe that, for both cases, the IIR scheme significantly outperforms the DIR scheme, and for both cases our analytical model remains accurate.

The performance of the DIR scheme with sparse conversion and the IIR scheme with no conversion is compared in Fig. 10.

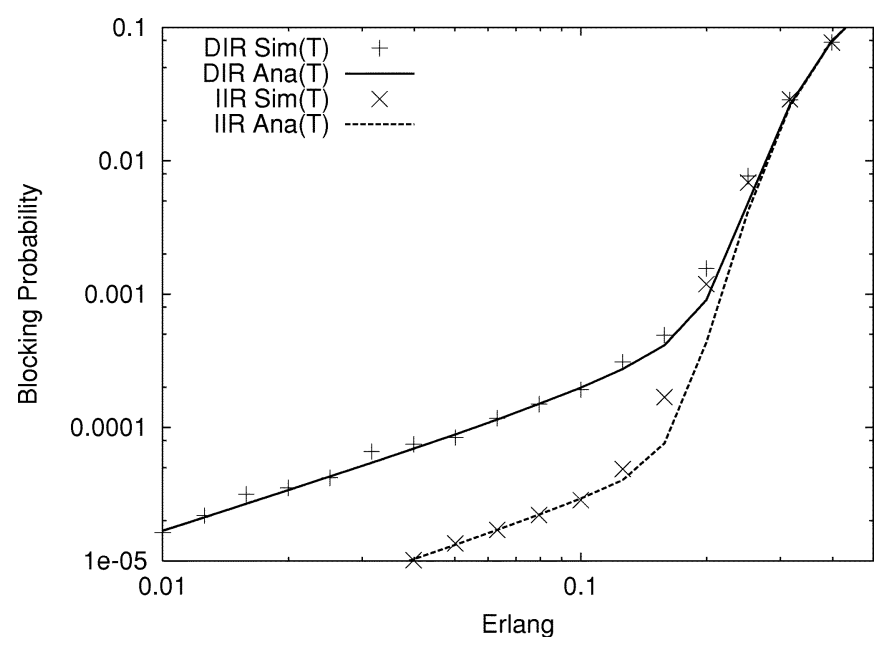

(a)

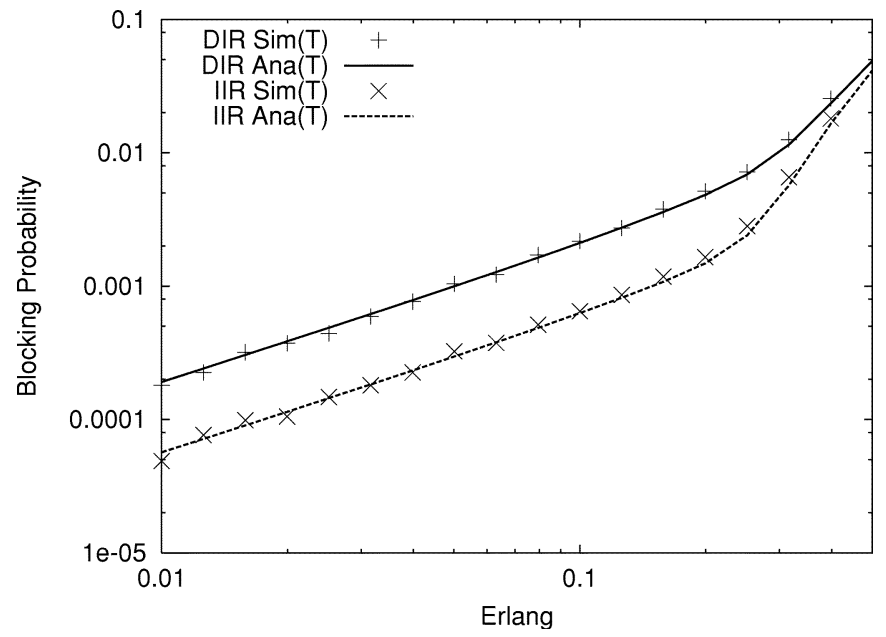

(b)

Fig. 9. DIR versus IIR scheme in other topologies with sparse wavelength conversion ( $\mathrm{C}=16, \mathrm{D}=0.1 \mathrm{~s}$ ). (a) 12-node-ring. (b) 25-node network.

We see that, although sparse wavelength conversion helps to reduce the blocking on both the forward and the backward directions (and actually helps the DIR scheme to achieve better performance than the IIR scheme in the forward direction), the overall performance of the IIR scheme is still better. This interesting result shows that we may expect to achieve better performance by using the new IIR scheme instead of expensive wavelength converters.

Finally, in Table I, we compare the control overhead of the IIR scheme and the DIR scheme, respectively. Specifically, whenever a control message arrives at, or is initiated by a node, we note that a message processing operation is needed at this node. Whenever a control messages passes through a link, we note that we have one more unit of control traffic loads. We record the total number of message processing operations, as well as all control traffic load, and we take into consideration all the connection, reservation, and release requests (including those for unsuccessful connections). Simulation results show that since the extra control overhead only comes from the fast reservation requests and the release requests when (and only when) the conflict occurs on the last link, the IIR scheme does not lead to significantly heavier control overhead compared with the DIR 


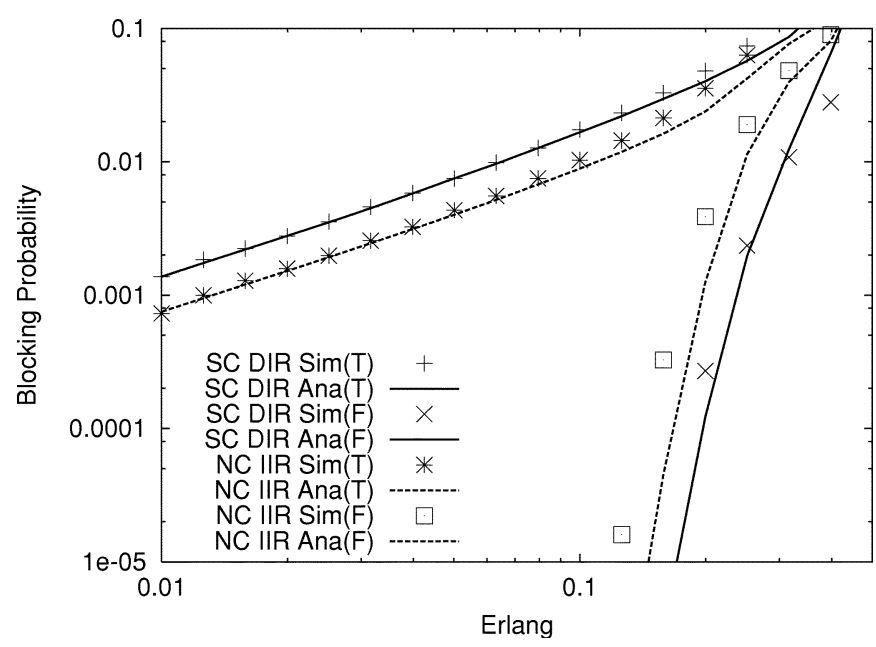

Fig. 10. DIR with sparse conversion versus IIR with no conversion in NSFNet $(\mathrm{C}=32, \mathrm{D}=0.1 \mathrm{~s})$.

TABLE I

Average Control Overhead of Each CONNECTION. (a) No CONVERSION (NSFNet, $C=16, D=1 \mathrm{~S}$ ).

(b) SPARSE CONVERSION (NSFNet, $C=16, D=1 \mathrm{~S}$ )

\begin{tabular}{c||c|c||c|c}
\hline Load (Erlang) & DIR(P*) & IIR(P) & DIR(T $\left.{ }^{\dagger}\right)$ & IIR(T) \\
\hline \hline 0.1 & 9.9 & 11.6 & 7.9 & 9.6 \\
\hline 0.5 & 8.6 & 10.1 & 6.7 & 8.2 \\
\hline 1.0 & 6.9 & 8.3 & 5.2 & 6.6 \\
\hline
\end{tabular}

(a)

\begin{tabular}{c||c|c||c|c}
\hline Load (Erlang) & DIR(P) & IIR(P) & DIR(T) & IIR(T) \\
\hline \hline 0.1 & 9.9 & 11.9 & 7.9 & 9.9 \\
\hline 0.5 & 8.9 & 10.8 & 7.0 & 8.9 \\
\hline 1.0 & 7.1 & 8.8 & 5.4 & 7.1 \\
\hline \hline
\end{tabular}

*: Number of messages processing operations; $\dagger$ : Number of units of control traffic loads.

(b)

scheme. Considering the significant performance improvements we could achieve by using the IIR scheme, the additional control overhead may well be justified. Finally, we found that the extra control overhead becomes slightly higher in networks with sparse wavelength conversion. This increase is caused by the extra segment reservation messages.

\section{CONCLUSION}

In this paper, we proposed a new framework for signaling schemes in distributed lightpath provisioning. By shortening the vulnerable period, we can significantly lower the blocking probabilities caused by outdated information, and reduce or even eliminate any over-reservation as well. As a first step of our research, we discussed two simple signaling cases within the framework, for networks with and without sparse wavelength conversion, respectively. Highly accurate analytical models were also developed to evaluate and confirm the most significant factors affecting the network performance. Analysis and simulation results demonstrate that the new scheme steadily and significantly outperforms the classic DIR scheme, at the cost of a reasonable amount of additional control overhead.

\section{APPENDIX}

\section{A. Calculation of Parameter $f_{i, j}$ and $f_{i, j \mid i, j^{\prime}}$}

To calculate $f_{i, j}$, we first define the following time parameters:

$t_{R} \quad$ denotes the round trip propagation delay between the source and the destination node of route $R$;

$\tau_{R}(j) \quad$ denotes the round trip propagation delay between the source node of $R$ and the downstream node of link $j$;

$t_{R}(j) \quad$ denotes the round trip propagation delay between the downstream node of link $j$ and the destination node of $R$. Therefore, $t_{R}=t_{R}(j)+\tau_{R}(j), \forall j$;

$\sigma_{R}(j) \quad$ denotes the round trip propagation delay between the downstream node of the link $j$ to the upstream node of the last link of segment $s$, provided that link $j$ is in $s$.

We define the reservation duration $t_{R}^{r}(j)$ as the duration from the moment that a channel on link $j$ is reserved to the moment that it becomes busy. In the classic DIR scheme, the reservation duration is

$$
t_{R}^{r}(j)=\tau_{R}(j)
$$

[see Fig. 1(a) and (b)]. On the other hand, in the IIR scheme, this duration becomes

$$
t_{R}^{r}(j)= \begin{cases}t_{R}, & j=j_{R}^{s} \\ t_{R}-\sigma_{R}(s), & j \neq j_{R}^{s}\end{cases}
$$

where $s$ is the segment that includes link $j$ and $j_{R}^{s}$ is the last link of segment $s$ (see Figs. 2 and 3). From the definition of $f_{i, j}$, we then have

$$
f_{i, j}=\sum_{m=i}^{C} q_{j \mid i}(m) \prod_{R^{\prime}: j \in R^{\prime}}\left(1-A_{R^{\prime}, j}\left(m, t_{R^{\prime}}^{r}(j)\right) \times \frac{i}{m}\right)
$$

where $R^{\prime}$ represents the route of any interfering lightpath; $q_{j \mid i}(m)$ denotes the probability that $m$ channels are idle on link $j$ given that a specific set of $i$ channels $(i \leq m)$ are idle on this link (see [16] for details); and

$$
A_{R^{\prime}, j}(m, t)=1-e^{-\lambda_{R^{\prime}, j}(m) t}
$$

denotes the probability that there is one connection request for $R^{\prime}$ arriving at link $j$ during time $t$.

The calculation of $f_{i, j \mid i, j^{\prime}}$ is nearly the same as that of $f_{i, j}$ except that $t_{R^{\prime}}^{r}(j)=2 \times t_{j}$ if $R^{\prime}$ also passes through link $j^{\prime}$.

\section{B. Calculation of Parameter $y_{s}$}

Parameter $y_{s}$ can be calculated as

$$
y_{s}\left(t_{1}\right)=1-\sum_{m=1}^{C} q_{j}(m)\left(1-y_{s \mid X_{j}=m}\left(t_{1}\right)\right)
$$

and

$$
y_{s \mid X_{j}=m}\left(t_{1}\right)=\prod_{R^{\prime}: j \in R^{\prime}}\left(1-\left(A_{R^{\prime}, j}\left(m, t_{1}\right)\right) \times \frac{1}{m}\right) .
$$

\section{REFERENCES}

[1] I. Chlamtac, A. Ganz, and G. Karmi, "Lightpath communications: A novel approach to high bandwidth optical WANs," IEEE Trans. Commun., vol. 40, pp. 1171-1182, July 1992. 
[2] J. M. H. Elmirghani and H. T. Mouftah, "Technologies and architectures for scalable dynamic dense WDM networks," IEEE Commun. Mag., vol. 38, pp. 58-66, Feb. 2000.

[3] J. Strand, A. L. Chiu, and R. Tkach, "Issues for routing in the optical layer," IEEE Commun. Mag., vol. 39, pp. 81-87, Feb. 2001.

[4] C. Qiao and M. Yoo, "Optical Burst Switch (OBS)—A new paradigm for an optical internet," J. High Speed Network, vol. 8, pp. 69-84, 1999.

[5] M. Duser and P. Bayvel, "Analysis of a dynamically wavelength-routed optical burst switched network architecture," J. Lightwave Technol., vol. 20, pp. 574-585, Apr. 2002.

[6] L. Berger, "Generalized multi-protocol label switching (GMPLS) signaling functional description," IETF, RFC 3471, Jan. 2003.

[7] —-, "Generalized multi-protocol label switching (GMPLS) signaling resource reservation protocol-traffic engineering (RSVP-TE) extensions," IETF, RFC 3473, Jan. 2003.

[8] P. Ashwood-Smith et al., "Generalized multi-protocol label switching (GMPLS) signaling constraint-based routed label distribution protocol (CR-LDP) extensions," IETF, RFC 3472, Jan. 2003.

[9] X. Yuan, R. Melhem, R. Gupta, Y. Mei, and C. Qiao, "Distributed control protocols for wavelength reservation and their performance evaluation," Photonic Network Commun., vol. 1, no. 3, pp. 207-218, 1999.

[10] J. M. H. Elmirghani and H. T. Mouftah, "All-optical wavelength conversion: Technologies and applications in DWDM networks," IEEE Commun. Mag., vol. 38, pp. 86-92, Mar. 2000.

[11] S. Subramaniam, M. Azizoglu, and A. K. Somani, "All-optical networks with sparse wavelength conversion," IEEE/ACM Trans. Network, vol. 4, no. 4, pp. 544-557, Aug. 1996.

[12] A. G. Stoica and A. Sengupta, "On a dynamic wavelength assignment algorithm for wavelength-routed all-optical networks," in Proc. SPIE/IEEE/ACM OptiComm 2000, Dallas, TX, Oct. 2000, pp. 211-222.

[13] A. Birman, "Computing approximate blocking probability for a class of all-optical networks," IEEE J. Select. Areas Commun., vol. 14, pp. 852-857, June 1996.

[14] A. Sridharan and K. N. Sivarajan, "Blocking in all-optical networks," in Proc. IEEE INFOCOM'O0, vol. 2, Tel Aviv, Israel, Mar. 2000, pp. 990-999.

[15] J. P. Jue and G. Xiao, "Analysis of blocking probability for connection management schemes in optical networks," in Proc. IEEE GLOBECOM'01, vol. 3, San Antonio, TX, Nov. 2001, pp. 1546-1550.

[16] K. Lu, G. Xiao, and I. Chlamtac, "Blocking analysis of dynamic lightpath establishment in wavelength-routed networks," in Proc. IEEE ICC'02, vol. 5, New York, Apr. 2002, pp. 2912-2916.

[17] K. Lu, J. P. Jue, T. Ozugur, G. Xiao, and I. Chlamtac, "Intermediate-node Initiated Reservation (IIR): A new signaling scheme for wavelengthrouted networks with sparse conversion," in Proc. IEEE ICC'03, Anchorage, AK, May 2003.

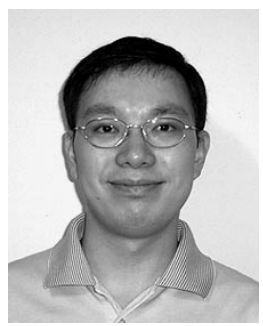

Kejie Lu (S'01) received the B.S. and M.S. degrees in telecommunications engineering from Beijing University of Posts and Telecommunications, Beijing, China, in 1994 and 1997, respectively. He is now working toward the Ph.D. degree in electrical engineering at the University of Texas at Dallas, Richardson.

His research interests include WDM optical networks, protocols design, and performance evaluation.

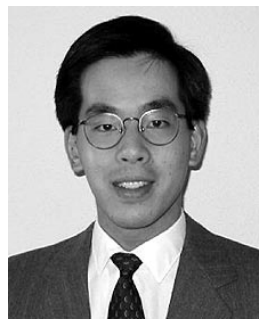

Jason P. Jue (M'99) received the B.S. degree in electrical engineering from the University of California, Berkeley, in 1990, the M.S. degree in electrical engineering from the University of California, Los Angeles in 1991, and the Ph.D. degree in computer engineering from the University of California, Davis, in 1999.

In 1999, he joined the faculty at the University of Texas at Dallas, Richardson, where he is currently an Assistant Professor of computer science. He is conducting research in the area of WDM optical networks, focusing on the design and analysis of optical network architectures and protocols.

Dr. Jue was a recipient of the National Science Foundation (NSF) Career Award in 2002

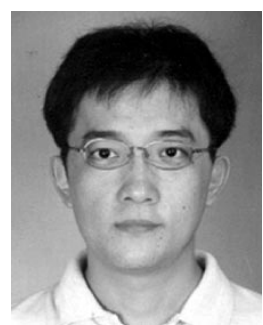

Gaoxi Xiao (M'99) received the B.S. and M.S. degrees in applied mathematics from Xidian University, Xi' an, China, in 1991 and 1994 respectively, and the Ph.D. degree from Department of Computing, the Hong Kong Polytechnic University, Hong Kong, China, in 1999.

From 1994 to 1995, he was with the Institute of Antenna and Electromagnetic Scattering, Xidian University. In 1999, he worked as a Postdoctoral Fellow in the Department of Electronic Engineering, Polytechnic University, Brooklyn, NY. From 1999 to 2001, he worked as a Visiting Scientist in the Center for Advanced Telecommunications Systems and Services (CATSS), University of Texas at Dallas, Richardson. Since October 2001, he has been an Assistant Professor with the Division of Communication Engineering, School of Electrical and Electronic Engineering, Nanyang Technological University, Singapore. His research interests include WDM optical networking, algorithm design and analysis, and IP over WDM.

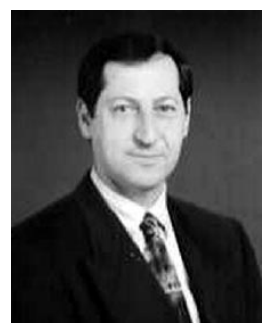

Imrich Chlamtac (F'93) received the Ph.D. degree in computer science from the University of Minnesota, Minneapolis.

Since 1997, he has been the Distinguished Chair in Telecommunications at the University of Texas at Dallas, Richardson, and holds the titles of the Sackler Professor at Tel-Aviv University, Tel-Aviv, Israel, The Bruno Kessler Honorary Professor at the University of Trento, Trento, Italy, and University Professor at the Technical University of Budapest, Budapest, Hungary. He has published over 300 papers in refereed journals and conferences, and is the coauthor of the first textbook on Local Area Networks (Lexington Books, 1981, 1982, 1984) and Mobile and Wireless Networks Protocols and Services (New York: Wiley, 2000). He is the founding Editor-in-Chief of the Wireless Networks (WINET), Mobile Networks and Applications (MONET), and Optical Networks Magazine (ONM) .

Dr. Chlamtac is a Fellow of the Association for Computing Machinery (ACM) Societies, a Fulbright Scholar, and an IEEE Distinguished Lecturer. He is the winner of the 2001 ACM Sigmobile Annual Award and the IEEE ComSoc TCPC 2002 Award for contributions to wireless and mobile networks, and of multiple best paper awards in wireless and optical networks.

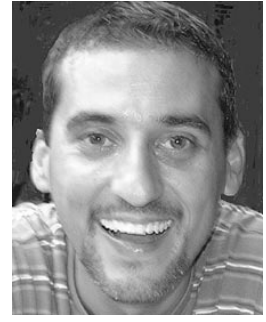

Timucin Ozugur (M'00) received the M.Sc. and B.Sc. degrees from Istanbul Technical University, Istanbul, Turkey, in 1995 and 1993, respectively, and the Ph.D. degree from Georgia Institute of Technology, Atlanta, in 2000, all in electrical engineering.

$\mathrm{He}$ is currently a Research Case Leader at the Research and Innovation Center, Alcatel, Plano, TX. Prior to his current position, he worked at Nortel Networks, Richardson, TX, IBM T. J. Watson Research Center, Yorktown Heights, NY, and Lucent Technologies-Bell Laboratories, Holmdel, NJ. He has been actively involved in several journals and conferences as a reviewer. He was the Guest Co-Editor of Optical Networks Magazine. He has published over 30 articles in journals and international conferences. He also holds one U.S. patent and several patent applications pending. His current research interests are mobile networking, optical networking, session initiation protocol, and enterprise applications.

Dr. Ozugar participated as a Local Chair in OPTICOMM 2003, and as a TPC Member in IPOM 2002, INFOCOM 2002, INFOCOM 2003, ICC 2003, and GLOBECOM 2003. He was the recipient of NATO Scholarship Award. 